In this paper we investigate the solution of boundary value problems on polygonal domains for elliptic partial differential equations. We observe that when the problems are formulated as the boundary integral equations of classical potential theory, the solutions are representable by series of elementary functions. In addition to being analytically perspicuous, the resulting expressions lend themselves to the construction of accurate and efficient numerical algorithms. The results are illustrated by a number of numerical examples.

Keywords: Boundary value problems, Potential theory, Corners, Singular solutions, Elliptic partial differential equations

\title{
On the solution of elliptic partial differential equations on regions with corners
}

\author{
K. Serkh ${ }^{\ddagger \diamond \oplus *}$, V. Rokhlin ${ }^{\ddagger \diamond \ominus}$,
}

August 12, 2015

^ This author's work was supported in part under ONR N00014-11-1-0718, ONR N0001414-1-0797

$\oplus$ This author's work was supported in part under ONR N00014-10-1-0570 and by the National Defense Science and Engineering Graduate Fellowship

$\ominus$ This author's work was supported in part under AFOSR FA-9550-09-1-0027

‡ Dept. of Mathematics, Yale University, New Haven CT 06511

* Corresponding author. Email: kirill.serkh@yale.edu 


\section{Introduction}

In classical potential theory, elliptic partial differential equations (PDEs) are reduced to integral equations by representing the solutions as single-layer or double-layer potentials on the boundaries of the regions. The densities of these potentials satisfy Fredholm integral equations of the second kind.

There are three essentially separate regimes in which such boundary integral equations have been studied. In the first regime, the boundary of the region is approximated by a smooth curve. It is known that if the curve is smooth, then the kernel of the integral equation is smooth as well (see, for example, [22]). The existence and uniqueness of the solution follows from Fredholm's theory, and the integral equations can be solved numerically using standard tools (see, for example, [12]).

In the second regime, the boundary of the region is approximated by a curve with perfectly sharp corners. In this regime, the kernel of the integral equation has singularities at the corners, and the existence and uniqueness of the solution in the $L^{2}$-sense is also known (see, for example, [24]). The behavior in the vicinity of the corners of the solutions of both the integral equations and of the underlying differential equation have been the subject of much study (see [26], [16] for representative examples). Comprehensive reviews of the literature can be found in (for example) [20], [11].

In the third regime, the assumptions on the boundary are of an altogether different nature. It might be a Lipschitz or Hölder continuous curve, or a fractal, etc. While during the last fifty years, such environments have been studied in great detail (see, for example, [14], [24], [4], [6], [5], [15], etc.), they are outside the scope of this paper.

This paper deals with the very special case of polygonal boundaries, and is based on several specific analytical observations. These observations are summarized in the following two subsections, and are discussed in detail in sections 4 and 5 .

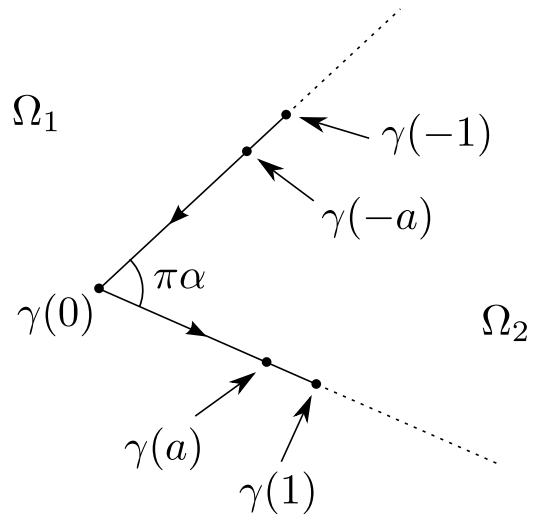

Figure 1: A wedge in $\mathbb{R}^{2}$ 


\subsection{The Neumann Case}

Suppose that $\gamma:[-1,1] \rightarrow \mathbb{R}^{2}$ is a wedge in $\mathbb{R}^{2}$ with a corner at $\gamma(0)$, and with interior angle $\pi \alpha$. Suppose further that $\gamma$ is parametrized by arc length, and let $\nu(t)$ denote the inward-facing unit normal to the curve $\gamma$ at $t$. Let $\Gamma$ denote the set $\gamma([-1,1])$. By extending the sides of the wedge to infinity, we divide $\mathbb{R}^{2}$ into two open sets $\Omega_{1}$ and $\Omega_{2}$ (see Figure 1).

Let $\phi: \mathbb{R}^{2} \backslash \Gamma \rightarrow \mathbb{R}$ denote the potential induced by a charge distribution on $\gamma$ with density $\rho:[-1,1] \rightarrow \mathbb{R}$. In other words, $\phi$ is defined by the formula

$$
\phi(x)=\int_{-1}^{1} \log (\|\gamma(t)-x\|) \rho(t) d t
$$

for all $x \in \mathbb{R}^{2} \backslash \Gamma$, where $\|\cdot\|$ denotes Euclidean distance. Suppose that $n$ is a positive integer, and let $\lceil x\rceil$ denote the smallest integer $n$ such that $n \geq x$, and $\lfloor x\rfloor$ denote the largest integer $n$ such that $n \leq x$, for all real $x$. Suppose further that $\rho$ is defined by the formula

$$
\rho(t)=\sum_{i=1}^{\lceil n / 2\rceil} b_{2 i-1}|t|^{\frac{2 i-1}{\alpha}-1}+\sum_{i=1}^{\lfloor n / 2\rfloor} b_{2 i} \operatorname{sgn}(t)|t|^{\frac{2 i}{\alpha}-1}+\sum_{i=1}^{\lceil n / 2\rceil} c_{2 i-1} \operatorname{sgn}(t)|t|^{\frac{2 i-1}{2-\alpha}-1}+\sum_{i=1}^{\lfloor n / 2\rfloor} c_{2 i}|t|^{\frac{2 i}{2-\alpha}-1},
$$

for all $-1 \leq t \leq 1$, where $b_{1}, b_{2}, \ldots, b_{n}$ and $c_{1}, c_{2}, \ldots, c_{n}$ are real numbers, and

$$
\operatorname{sgn}(x)= \begin{cases}-1 & \text { if } x<0 \\ 0 & \text { if } x=0 \\ 1 & \text { if } x>0\end{cases}
$$

for all real $x$. Suppose finally that $g:[-1,1] \rightarrow \mathbb{R}$ is defined by the formula

$$
g(t)=\lim _{\substack{x \rightarrow \gamma(t) \\ x \in \Omega_{1}}} \frac{\partial \phi(x)}{\partial \nu(t)}
$$

for all $-1 \leq t \leq 1$, i.e. $g$ is the limit of the normal derivative of integral (1) when $x$ approaches the point $\gamma(t)$ from outside. Then $g$ is smooth on each of the intervals $[-a, 0],[0, a]$ for any $0<a<1$ (see Figure 1 ). In other words, given a charge distribution $\rho$ of the form (2), the normal derivative of the resulting potential (1) on $\Gamma$ (in the sense (4)) is smooth.

Conversely, for any sufficiently smooth $g$, there exists a charge distribution $\rho$ of the form (2) such that the normal derivative of the resulting potential (1) on $\Gamma$ is equal to $g$, to high precision. 


\subsection{The Dirichlet Case}

Suppose that $\gamma:[-1,1] \rightarrow \mathbb{R}^{2}$ is a wedge in $\mathbb{R}^{2}$ with a corner at $\gamma(0)$, and with interior angle $\pi \alpha$. Suppose further that $\gamma$ is parametrized by arc length, and let $\nu(t)$ denote the inward-facing unit normal to the curve $\gamma$ at $t$. Let $\Gamma$ denote the set $\gamma([-1,1])$. By extending the sides of the wedge to infinity, we divide $\mathbb{R}^{2}$ into two open sets $\Omega_{1}$ and $\Omega_{2}$ (see Figure 1).

Let $\phi: \mathbb{R}^{2} \backslash \Gamma \rightarrow \mathbb{R}$ denote the potential induced by a dipole distribution on $\gamma$ with density $\rho:[-1,1] \rightarrow \mathbb{R}$. In other words, $\phi$ is defined by the formula

$$
\phi(x)=\int_{-1}^{1} \frac{\langle\nu(t), \gamma(t)-x\rangle}{\|\gamma(t)-x\|^{2}} \rho(t) d t
$$

for all $x \in \mathbb{R}^{2} \backslash \Gamma$, where $\langle\cdot, \cdot\rangle$ denotes the inner product and $\|\cdot\|$ denotes Euclidean distance. Suppose that $n$ is a positive integer, and let $\lceil x\rceil$ denote the smallest integer $n$ such that $n \geq x$, and $\lfloor x\rfloor$ denote the largest integer $n$ such that $n \leq x$, for all real $x$. Suppose further that $\rho$ is defined by the formula

$$
\rho(t)=\sum_{i=1}^{\lceil n / 2\rceil} b_{2 i-1}|t|^{\frac{2 i-1}{\alpha}}+\sum_{i=0}^{\lfloor n / 2\rfloor} b_{2 i} \operatorname{sgn}(t)|t|^{\frac{2 i}{\alpha}}+\sum_{i=1}^{\lceil n / 2\rceil} c_{2 i-1} \operatorname{sgn}(t)|t|^{\frac{2 i-1}{2-\alpha}}+\sum_{i=0}^{\lfloor n / 2\rfloor} c_{2 i}|t|^{\frac{2 i}{2-\alpha}}
$$

for all $-1 \leq t \leq 1$, where $b_{0}, b_{1}, \ldots, c_{n}$ and $c_{0}, c_{1}, \ldots, c_{n}$ are real numbers, and $\operatorname{sgn}(x)$ is defined by (3). Suppose finally that $g:[-1,1] \rightarrow \mathbb{R}$ is defined by the formula

$$
g(t)=\lim _{\substack{x \rightarrow \gamma(t) \\ x \in \Omega_{2}}} \phi(x)
$$

for all $-1 \leq t \leq 1$, i.e. $g$ is the limit of integral (5) when $x$ approaches the point $\gamma(t)$ from inside. Then $g$ is smooth on each of the intervals $[-a, 0],[0, a]$ for any $0<a<1$ (see Figure 1). In other words, given a dipole distribution $\rho$ of the form (6), the resulting potential (5) on $\Gamma$ (in the sense (7)) is smooth.

Conversely, for any sufficiently smooth $g$, there exists a dipole distribution $\rho$ of the form (6) such that the resulting potential (5) on $\Gamma$ is equal to $g$, to high precision.

\subsection{The Procedure}

Recently, progress has been made in solving the boundary integral equations of potential theory numerically (see, for example, [13], [3]). Most such schemes use nested quadratures to resolve the corner singularities. However, the explicit representations (2), (6) lead to alternative numerical algorithms for the solution of the integral equations of potential 
theory. More specifically, we use these representations to construct purpose-made discretizations which accurately represent the associated boundary integral equations (see, for example, [19], [17], [25]). Once these discretizations are available, the equations can be solved using the Nyström method combined with standard tools. We observe that the condition numbers of the resulting discretized linear systems closely approximate the condition numbers of the underlying physical problems.

Observation 1.1 While the analysis in this paper applies only to polygonal domains, a similar analysis carries over to curved domains with corners. A paper containing the analysis, as well as the corresponding numerical algorithms and numerical examples, is in preparation.

Observation 1.2 In the examples in this paper, the discretized boundary integral equations are solved in a straightforward way using standard tools. However, if needed, such equations can be solved much more rapidly using, for example, [10].

Remark 1.3 Due to the detailed analysis in this paper, the CPU time requirements of the resulting algorithms are almost independent of the requested precision. Thus, in all the examples in this paper, the boundary integral equations are solved to essentially full double precision.

The structure of the paper is as follows. In Section 2, we introduce the necessary mathematical preliminaries. Section 3 contains the primary analytical tools of the paper. In sections 4 and 5, we investigate the Neumann and Dirichlet cases respectively. In Section 6, we briefly describe a numerical algorithm and provide several numerical examples.

\section{Mathematical Preliminaries}

\subsection{Boundary Value Problems}

Suppose that $\gamma:[0, L] \rightarrow \mathbb{R}^{2}$ is a simple closed curve of length $L$ with $n$ corners at the points $0=s_{1}<s_{2}<\ldots<s_{n}<s_{n+1}=L$. Suppose further that $\gamma$ is parameterized by arc length and oriented counterclockwise. We denote the interior of $\gamma$ by $\Omega$, and denote the boundary of $\Omega$ by $\Gamma$. Suppose that $\gamma$ is analytic on the intervals $\left(s_{i}, s_{i+1}\right)$ for each $i=1,2, \ldots, n$, and that the interior angle at any corner of $\gamma$ is between 0 and $2 \pi$. We denote the normalized internal normal to $\gamma$ at $t \in[0, L]$ by $\nu(t)$. Letting $g$ be a function $[0, L] \rightarrow \mathbb{R}$, we will consider the following problems. 


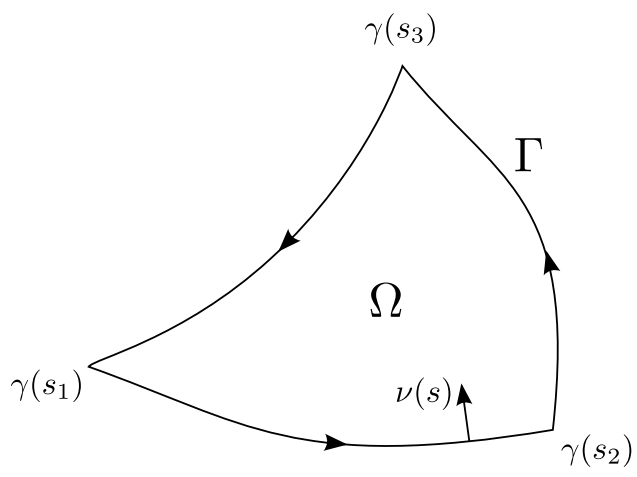

Figure 2: A curve in $\mathbb{R}^{2}$

Exterior Neumann problem: find a function $\phi: \mathbb{R}^{2} \backslash \Omega \rightarrow \mathbb{R}$ such that

$$
\begin{array}{lr}
\nabla^{2} \phi(x)=0 & \text { for } x \in \mathbb{R}^{2} \backslash \bar{\Omega}, \\
\lim _{\substack{x \rightarrow \gamma(t) \\
x \in \mathbb{R}^{2} \backslash \bar{\Omega}}} \frac{\partial \phi(x)}{\partial \nu(t)}=g(t) & \text { for } t \in[0, L] .
\end{array}
$$

Interior Dirichlet problem: find a function $\phi: \Omega \rightarrow \mathbb{R}$ such that

$$
\begin{array}{lr}
\nabla^{2} \phi(x)=0 & \text { for } x \in \Omega, \\
\lim _{\substack{x \rightarrow \gamma(t) \\
x \in \Omega}} \phi(x)=g(t) & \text { for } t \in[0, L] .
\end{array}
$$

For every $g \in L^{2}([0, L])$, the exterior Neumann problem and the interior Dirichlet problem have unique solutions (see, for example, [14]).

\subsection{Integral Equations of Potential Theory}

In classical potential theory, boundary value problems are solved by representing the function $\phi$ by integrals of potentials over the boundary. The potential of a unit charge located at $x_{0} \in \mathbb{R}^{2}$ is the function $\psi_{x_{0}}^{0}: \mathbb{R}^{2} \backslash x_{0} \rightarrow \mathbb{R}$, defined via the formula

$$
\psi_{x_{0}}^{0}(x)=\log \left(\left\|x-x_{0}\right\|\right),
$$

for all $x \in \mathbb{R}^{2} \backslash x_{0}$, where $\|\cdot\|$ denotes Euclidean distance. The potential of a unit dipole located at $x_{0} \in \mathbb{R}^{2}$ and oriented in direction $h \in \mathbb{R}^{2},\|h\|=1$, is the function $\psi_{x_{0}, h}^{1}: \mathbb{R}^{2} \backslash x_{0} \rightarrow \mathbb{R}$, defined via the formula

$$
\psi_{x_{0}, h}^{1}(x)=\frac{\left\langle h, x_{0}-x\right\rangle}{\left\|x_{0}-x\right\|^{2}},
$$


for all $x \in \mathbb{R}^{2} \backslash x_{0}$, where $\langle\cdot, \cdot\rangle$ denotes the inner product.

The potential created by a charge distribution with density $\rho:[0, L] \rightarrow \mathbb{R}$ on $\Gamma$ is given by the formula

$$
\phi(x)=\int_{0}^{L} \psi_{\gamma(t)}^{0}(x) \rho(t) d t,
$$

for any $x \in \mathbb{R}^{2} \backslash \Gamma$, and is called a single layer potential. The potential created by a dipole distribution with density $\rho:[0, L] \rightarrow \mathbb{R}$ on $\Gamma$ is given by the formula

$$
\phi(x)=\int_{0}^{L} \psi_{\gamma(t), \nu(t)}^{1}(x) \rho(t) d t,
$$

for any $x \in \mathbb{R}^{2} \backslash \Gamma$, and is called a double layer potential.

\subsubsection{Reduction of Boundary Value Problems to Integral Equations}

The following two theorems reduce the boundary value problems of Section 2.1 to boundary integral equations. They are found in, for example, [24].

Theorem 2.1 Suppose that $\rho \in L^{2}([0, L])$. Suppose further that $g:[0, L] \rightarrow \mathbb{R}$ is defined by the formula

$$
g(s)=-\pi \rho(s)+\int_{0}^{L} \psi_{\gamma(s), \nu(s)}^{1}(\gamma(t)) \rho(t) d t,
$$

for any $s \in[0, L]$. Then $g$ is in $L^{2}([0, L])$, and the solution $\phi$ to the exterior Neumann problem with right hand side $g$ is given by (14). Moreover, for any $g \in L^{2}([0, L])$, equation (16) has a unique solution $\rho \in L^{2}([0, L])$.

Theorem 2.2 Suppose that $\rho \in L^{2}([0, L])$. Suppose further that $g:[0, L] \rightarrow \mathbb{R}$ is defined by the formula

$$
g(s)=-\pi \rho(s)+\int_{0}^{L} \psi_{\gamma(t), \nu(t)}^{1}(\gamma(s)) \rho(t) d t,
$$

for any $s \in[0, L]$. The $g$ is in $L^{2}([0, L])$, and the solution $\phi$ to the interior Dirichlet problem with right hand side $g$ is given by (15). Moreover, for any $g \in L^{2}([0, L])$, equation (17) has a unique solution $\rho \in L^{2}([0, L])$.

Observation 2.1 Equation (16) is the adjoint of equation (17).

Observation 2.2 Suppose that the curve $\gamma:[0, L] \rightarrow \mathbb{R}^{2}$ is not closed. We observe that if $\rho \in L^{2}([0, L])$, and $g$ is defined by either (16) or (17), then $g \in L^{2}([0, L])$. Moreover, if $g \in L^{2}([0, L])$, then both equations (16) and (17) have unique solutions $\rho \in L^{2}([0, L])$. 


\subsubsection{Properties of the Kernels of Equations (16) and (17)}

The following theorem shows that if a curve $\gamma$ is has $k$ continuous derivatives, where $k \geq 2$, then the kernels of equations (16) and (17) have $k-2$ continuous derivatives. It is found in, for example, [7].

Theorem 2.3 Suppose that $\gamma:[0, L] \rightarrow \mathbb{R}^{2}$ is a curve in $\mathbb{R}^{2}$ that is parameterized by arc length. Suppose further that $k \geq 2$ is an integer. If $\gamma$ is $C^{k}$ on a neighborhood of a point $s$, where $0<s<L$, then

$$
\begin{aligned}
& \psi_{\gamma(s), \nu(s)}^{1}(\gamma(t)), \\
& \psi_{\gamma(t), \nu(t)}^{1}(\gamma(s)),
\end{aligned}
$$

are $C^{k-2}$ functions of $t$ on a neighborhood of s. Furthermore,

$$
\lim _{t \rightarrow s} \psi_{\gamma(s), \nu(s)}^{1}(\gamma(t))=\lim _{t \rightarrow s} \psi_{\gamma(t), \nu(t)}^{1}(\gamma(s))=-\frac{1}{2} k(s),
$$

where $k:[0, L] \rightarrow \mathbb{R}$ is the signed curvature of $\gamma$.

Remark 2.3 Suppose that the curve $\gamma:[0, L] \rightarrow \mathbb{R}^{2}$ is analytic on a neighborhood of a point $s$, where $0<s<L$. Then (18) and (19) are analytic functions of $t$ on a neighborhood of $s$.

When the curve $\gamma$ is a wedge, the kernels of equations (16) and (17) have a particularly simple form, which is given by the following lemma.

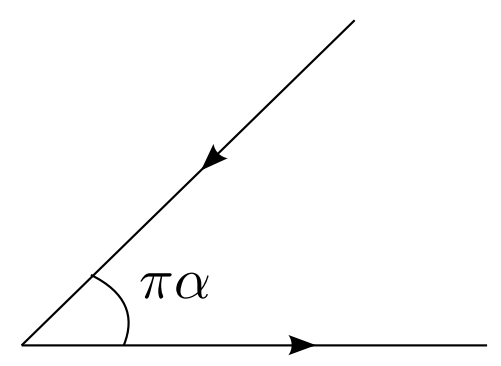

Figure 3: A wedge in $\mathbb{R}^{2}$

Lemma 2.4 Suppose $\gamma:[-1,1] \rightarrow \mathbb{R}^{2}$ is defined by the formula

$$
\gamma(t)= \begin{cases}-t \cdot(\cos (\pi \alpha), \sin (\pi \alpha)) & \text { if }-1 \leq t<0 \\ (t, 0) & \text { if } 0 \leq t \leq 1\end{cases}
$$


shown in Figure 3. Then, for all $0<s \leq 1$,

$$
\psi_{\gamma(s), \nu(s)}^{1}(\gamma(t))= \begin{cases}\frac{t \sin (\pi \alpha)}{s^{2}+t^{2}+2 s t \cos (\pi \alpha)} & \text { if }-1 \leq t<0, \\ 0 & \text { if } 0 \leq t \leq 1,\end{cases}
$$

and, for all $-1 \leq s<0$,

$$
\psi_{\gamma(s), \nu(s)}^{1}(\gamma(t))= \begin{cases}0 & \text { if }-1 \leq t<0 \\ \frac{-t \sin (\pi \alpha)}{s^{2}+t^{2}+2 s t \cos (\pi \alpha)} & \text { if } 0 \leq t \leq 1\end{cases}
$$

Corollary 2.5 Identities (22) and (23) remain valid after any rotation or translation of the curve $\gamma$ in $\mathbb{R}^{2}$.

Corollary 2.6 When the curve $\gamma$ is a straight line, $\psi_{\gamma(s), \nu(s)}^{1}(\gamma(t))=0$ for all $-1 \leq s \leq 1$ and $-1 \leq t \leq 1$.

\section{Analytical Apparatus}

The elementary theorem 3.4 in this section is the primary analytical tool of this paper.

The following theorem provides the value of a certain integral. It is found in, for example, [9], Section 3.252, formula 12.

Theorem 3.1 Suppose that $-1<\mu<1$ and $0<\alpha<2$ are real numbers. Then

$$
\int_{0}^{\infty} \frac{x^{\mu} \sin (\pi \alpha)}{a^{2}-2 a x \cos (\pi \alpha)+x^{2}} d x=\pi a^{\mu-1} \frac{\sin (\mu \pi(1-\alpha))}{\sin (\mu \pi)},
$$

for all $a>0$.

The following lemma gives the Taylor series of a certain rational function.

Lemma 3.2 Suppose that $-1<p<1$ and $x$ are real numbers. Then

$$
\frac{p \sin (x)}{1-2 p \cos (x)+p^{2}}=\sum_{n=1}^{\infty} p^{n} \sin (n x) \text {. }
$$


Proof. Let $-1<p<1$ and $x$ be real numbers. Then

$$
\begin{aligned}
& \sum_{n=1}^{\infty} p^{n} \sin (n x)=\operatorname{Im}\left(\sum_{n=0}^{\infty} p^{n} e^{i n x}\right)=\operatorname{Im}\left(\frac{1}{1-p e^{i x}}\right) \\
& =\operatorname{Im}\left(\frac{1-p e^{-i x}}{1-2 p \cos (x)+p^{2}}\right) \\
& =\frac{p \sin (x)}{1-2 p \cos (x)+p^{2}} .
\end{aligned}
$$

The following lemma evaluates the integral in (24) when it is taken from 0 to 1 instead of from 0 to $\infty$.

Lemma 3.3 Suppose that $-1<\mu<1$ and $0<\alpha<2$ are real numbers. Then

$$
\int_{0}^{1} \frac{x^{\mu} \sin (\pi \alpha)}{a^{2}-2 a x \cos (\pi \alpha)+x^{2}} d x=\pi a^{\mu-1} \frac{\sin (\mu \pi(1-\alpha))}{\sin (\mu \pi)}+\sum_{k=0}^{\infty} \frac{\sin ((k+1) \pi \alpha)}{\mu-k-1} a^{k}
$$

for all $0<a<1$.

Proof. Suppose that $0<a<1$. Clearly,

$$
\int_{1}^{\infty} \frac{x^{\mu} \sin (\pi \alpha)}{a^{2}-2 a x \cos (\pi \alpha)+x^{2}} d x=\int_{1}^{\infty} \frac{x^{\mu-1}}{a} \cdot \frac{\left(\frac{a}{x}\right) \sin (\pi \alpha)}{\left(\frac{a}{x}\right)^{2}-2\left(\frac{a}{x}\right) \cos (\pi \alpha)+1} d x .
$$

Since $\frac{a}{x}<1$ for all $x \geq 1$, by Lemma 3.2 we observe that

$$
\int_{1}^{\infty} \frac{x^{\mu} \sin (\pi \alpha)}{a^{2}-2 a x \cos (\pi \alpha)+x^{2}} d x=\int_{1}^{\infty} \frac{x^{\mu-1}}{a} \sum_{n=1}^{\infty} \frac{a^{n} \sin (n \pi \alpha)}{x^{n}} d x .
$$

Interchanging the order of integration and summation, we further observe that

$$
\begin{aligned}
& \int_{1}^{\infty} \frac{x^{\mu} \sin (\pi \alpha)}{a^{2}-2 a x \cos (\pi \alpha)+x^{2}} d x=\sum_{n=1}^{\infty} \int_{1}^{\infty} \frac{a^{n-1} \sin (n \pi \alpha)}{x^{n-\mu+1}} d x \\
& =-\sum_{n=1}^{\infty} \frac{a^{n-1} \sin (n \pi \alpha)}{\mu-n} \\
& =-\sum_{k=0}^{\infty} \frac{\sin ((k+1) \pi \alpha)}{\mu-k-1} a^{k} .
\end{aligned}
$$


Combining (24) and (30), we find that

$$
\int_{0}^{1} \frac{x^{\mu} \sin (\pi \alpha)}{a^{2}-2 a x \cos (\pi \alpha)+x^{2}} d x=\pi a^{\mu-1} \frac{\sin (\mu \pi(1-\alpha))}{\sin (\mu \pi)}+\sum_{k=0}^{\infty} \frac{\sin ((k+1) \pi \alpha)}{\mu-k-1} a^{k}
$$

for all $0<a<1$.

The following theorem is the primary analytical tool of this paper.

A simple analytic continuation argument shows that identity (27) in lemma 3.3 is also true for all complex $\mu$ such that $\operatorname{Re}(\mu)>-1$ and $\mu \neq 1,2,3, \ldots$. This observation is summarized by the following theorem.

Theorem 3.4 Suppose that $0<\alpha<2$ is a real number and $\mu$ is complex, so that $\operatorname{Re} \mu>-1$ and $\mu \neq 1,2,3, \ldots$ Then

$$
\int_{0}^{1} \frac{x^{\mu} \sin (\pi \alpha)}{a^{2}-2 a x \cos (\pi \alpha)+x^{2}} d x=\pi a^{\mu-1} \frac{\sin (\mu \pi(1-\alpha))}{\sin (\mu \pi)}+\sum_{k=0}^{\infty} \frac{\sin ((k+1) \pi \alpha)}{\mu-k-1} a^{k}
$$

for all $0<a<1$.

Proof. Suppose that $0<a<1$. We observe that the right and left hand sides of identity (27) are both analytic functions of $\mu$, for all $\mu$ such that $\operatorname{Re}(\mu)>-1$ and $\mu \neq 1,2,3, \ldots$ Therefore, by analytic continuation, it follows that identity (27) holds for all complex $\mu$ such that $\operatorname{Re}(\mu)>-1$ and $\mu \neq 1,2,3, \ldots$

The following lemma states that a certain series converges.

Lemma 3.5 Suppose that $m$ is a positive integer and $0<\alpha<2$ is a real number. Then

$$
\sum_{n=1}^{\infty} \frac{\sin (\pi n \alpha)}{m-n \alpha}<\infty
$$

Proof. We observe that

$$
\frac{1}{n \alpha-m} \geq \frac{1}{(n+1) \alpha-m}>0
$$

for all positive integers $n$ such that $n>m / \alpha$. Moreover,

$$
\lim _{n \rightarrow \infty} \frac{1}{n \alpha-m}=0
$$


We also observe that, for any positive integer $N$,

$$
\begin{aligned}
& \left|\sum_{n=1}^{N} \sin (\pi n \alpha)\right| \leq\left|\sum_{n=0}^{N} e^{i n \alpha}\right|=\left|\frac{1-e^{i(N+1) \alpha}}{1-e^{i \alpha}}\right| \\
& \leq\left|\frac{e^{i(N+1) \alpha / 2}}{e^{i \alpha / 2}} \cdot \frac{e^{-i(N+1) \alpha / 2}-e^{i(N+1) \alpha / 2}}{e^{-i \alpha / 2}-e^{i \alpha / 2}}\right| \\
& =2\left|\frac{\cos ((N+1) \alpha / 2)}{\cos (\alpha / 2)}\right| \\
& \leq \frac{2}{|\cos (\alpha / 2)|} .
\end{aligned}
$$

Hence, (33) follows by Dirichlet's test for the convergence of a series (see, for example, [2]).

The following theorem states that a certain Taylor series converges and is bounded on the interval $[0,1]$.

Theorem 3.6 Suppose that $m$ and $k$ are positive integers and $0<\alpha<2$ is a real number. Suppose further that $\phi$ is a function $[0,1] \rightarrow \mathbb{R}$ defined by the formula

$$
\phi(t)=\sum_{n=k}^{\infty} \frac{\sin (\pi n \alpha)}{m-n \alpha} t^{n-k}
$$

for all $0 \leq t \leq 1$. Then $\phi$ is well defined and bounded on the interval $[0,1]$.

Proof. We observe that

$$
\left|\frac{\sin (\pi n \alpha)}{m-n \alpha}\right|=\left|\frac{\sin (\pi(m-n \alpha))}{m-n \alpha}\right|=\pi\left|\frac{\sin (\pi(m-n \alpha))}{\pi(m-n \alpha)}\right| \leq \pi,
$$

for all positive integers $n$. Therefore,

$$
\sum_{n=k}^{\infty} \frac{\sin (\pi n \alpha)}{m-n \alpha} t^{n-k}<\infty
$$

for all $0 \leq t<1$. By Lemma 3.5,

$$
\sum_{n=k}^{\infty} \frac{\sin (\pi n \alpha)}{m-n \alpha}<\infty
$$


so $\phi$ is well defined on $[0,1]$. Furthermore, by Abel's theorem for power series (see, for example, [2]),

$$
\lim _{\substack{t \rightarrow 1 \\ t<1}} \sum_{n=k}^{\infty} \frac{\sin (\pi n \alpha)}{m-n \alpha} t^{n-k}=\sum_{n=k}^{\infty} \frac{\sin (\pi n \alpha)}{m-n \alpha},
$$

so $\phi$ is continuous on the interval $[0,1]$. Therefore, $\phi$ is bounded on $[0,1]$.

The following theorem states that a certain matrix is nonsingular.

Theorem 3.7 Suppose that $0<\alpha<2$ is a real number. Suppose further that $A(\alpha)$ is an $n \times n$ matrix defined via the formula

$$
A_{i, j}(\alpha)= \begin{cases}\alpha \cdot \frac{\sin (\pi \alpha i)}{2 j-1-\alpha i} & \text { if } i \text { is odd } \\ (2-\alpha) \cdot \frac{\sin (\pi \alpha i)}{2 j-(2-\alpha) i} & \text { if } i \text { is even }\end{cases}
$$

where $1 \leq i, j \leq n$ are integers. Then $A(\alpha)$ is nonsingular for all but a finite number of $0<\alpha<2$.

Proof. We observe that the functions

$$
\begin{aligned}
& \alpha \cdot \frac{\sin (\pi \alpha i)}{2 j-1-\alpha i}, \\
& (2-\alpha) \cdot \frac{\sin (\pi \alpha i)}{2 j-(2-\alpha) i},
\end{aligned}
$$

are entire functions of $\alpha$, where $1 \leq i, j \leq n$ are integers. Therefore, $\operatorname{det}(A(\alpha))$ is an entire function of $\alpha$. We also observe that

$$
A(1)=\pi I
$$

where $I$ is the identity matrix, from which it follows that

$$
\operatorname{det}(A(1))=\pi
$$

Since $\operatorname{det}(A(\alpha))$ is not identically zero and the interval $[0,2]$ is compact, it follows that $\operatorname{det}(A(\alpha))$ is equal to 0 at no more than a finite number of points in $[0,2]$. Hence, $A(\alpha)$ is nonsingular for all but a finite number of points $0<\alpha<2$. 


\section{Analysis of the Integral Equation: the Neumann Case}

Suppose that the curve $\gamma:[-1,1] \rightarrow \mathbb{R}^{2}$ is a wedge defined by (21) with interior angle $\pi \alpha$, where $0<\alpha<2$ (see Figure 3 ). Let $g$ be a function in $L^{2}([-1,1])$, and suppose that $\rho \in L^{2}([-1,1])$ solves the equation

$$
-\pi \rho(s)+\int_{-1}^{1} \psi_{\gamma(s), \nu(s)}^{1}(\gamma(t)) \rho(t) d t=g(s),
$$

for all $s \in[-1,1]$.

In this section, we will analyze this boundary integral equation, which is well-posed even though the curve $\gamma$ is open (see Observation 2.2). In Section 4.1 we investigate the behavior of (47) for functions $\rho \in L^{2}([-1,1])$ of the forms

$$
\begin{aligned}
& \rho(t)=|t|^{\mu-1}, \\
& \rho(t)=\operatorname{sgn}(t)|t|^{\mu-1},
\end{aligned}
$$

where $\mu>\frac{1}{2}$ is a real number and

$$
\operatorname{sgn}(x)= \begin{cases}-1 & \text { if } x<0, \\ 0 & \text { if } x=0, \\ 1 & \text { if } x>0,\end{cases}
$$

for all real $x$. If identities (22) and (23) are substituted into (47) and $\rho$ has the forms (48) and (49), then for most values of $\mu$ the resulting $g$ is singular. In Section 4.2, we observe that for certain $\mu$, the function $g$ is smooth. In Section 4.3, we fix $g$ and view (47) as an integral equation in $\rho$. We then observe that for certain classes of functions $g$, the solution $\rho$ is representable by a series of functions of the forms (48) and (49).

\subsection{Integral Equations Near a Corner}

The following lemma uses a symmetry argument to reduce (47) from an integral equation on the interval $[-1,1]$ to two independent integral equations on the interval $[0,1]$.

Theorem 4.1 Suppose that $\rho$ is a function in $L^{2}([-1,1])$ and that $g \in L^{2}([-1,1])$ is given by (47). Suppose further that even functions $g_{e}, \rho_{e} \in L^{2}([-1,1])$ are defined via the formulas

$$
\begin{aligned}
& g_{e}(s)=\frac{1}{2}(g(s)+g(-s)), \\
& \rho_{e}(s)=\frac{1}{2}(\rho(s)+\rho(-s)) .
\end{aligned}
$$


Then

$$
g_{e}(s)=-\pi \rho_{e}(s)-\int_{0}^{1} \frac{t \sin (\pi \alpha)}{s^{2}+t^{2}-2 s t \cos (\pi \alpha)} \rho_{e}(t) d t,
$$

for all $0<s \leq 1$.

Likewise, suppose that odd functions $g_{o}, \rho_{o} \in L^{2}([-1,1])$ are defined via the formulas

$$
\begin{aligned}
& g_{o}(s)=\frac{1}{2}(g(s)-g(-s)), \\
& \rho_{o}(s)=\frac{1}{2}(\rho(s)-\rho(-s)) .
\end{aligned}
$$

Then

$$
g_{o}(s)=-\pi \rho_{o}(s)+\int_{0}^{1} \frac{t \sin (\pi \alpha)}{s^{2}+t^{2}-2 s t \cos (\pi \alpha)} \rho_{o}(t) d t,
$$

for all $0<s \leq 1$.

Proof. By Lemma 2.3,

$$
g(s)=-\pi \rho(s)-\int_{0}^{1} \frac{t \sin (\pi \alpha)}{s^{2}+t^{2}+2 s t \cos (\pi \alpha)} \rho(t) d t,
$$

for all $-1 \leq s<0$, and

$$
g(s)=-\pi \rho(s)+\int_{-1}^{0} \frac{t \sin (\pi \alpha)}{s^{2}+t^{2}+2 s t \cos (\pi \alpha)} \rho(t) d t,
$$

for all $0<s \leq 1$. Therefore,

$$
g(-s)=-\pi \rho(-s)-\int_{0}^{1} \frac{t \sin (\pi \alpha)}{s^{2}+t^{2}-2 s t \cos (\pi \alpha)} \rho(t) d t
$$

for all $0<s \leq 1$, and

$$
g(s)=-\pi \rho(s)-\int_{0}^{1} \frac{t \sin (\pi \alpha)}{s^{2}+t^{2}-2 s t \cos (\pi \alpha)} \rho(-t) d t
$$

for all $0<s \leq 1$.

Adding equations (59) and (60), we observe that

$$
g_{e}(s)=-\pi \rho_{e}(s)-\int_{0}^{1} \frac{t \sin (\pi \alpha)}{s^{2}+t^{2}-2 s t \cos (\pi \alpha)} \rho_{e}(t) d t,
$$


for all $0<s \leq 1$.

Likewise, subtracting equation (59) from equation (60), we observe that

$$
g_{o}(s)=-\pi \rho_{o}(s)+\int_{0}^{1} \frac{t \sin (\pi \alpha)}{s^{2}+t^{2}-2 s t \cos (\pi \alpha)} \rho_{o}(t) d t
$$

for all $0<s \leq 1$.

\subsection{The Singularities in the Solution of Equation (47)}

In this section we observe that for certain functions $\rho$, the functions $g_{e}$ and $g_{o}$ defined by (51) and (54) are representable by convergent Taylor series on $[0,1]$.

\subsubsection{The Even Case}

Suppose that $\rho \in L^{2}([-1,1])$ is an even function, and suppose that $g \in L^{2}([-1,1])$ is defined by (47). By Theorem 4.1, $g$ is also even and

$$
g(s)=-\pi \rho(s)-\int_{0}^{1} \frac{t \sin (\pi \alpha)}{s^{2}+t^{2}-2 s t \cos (\pi \alpha)} \rho(t) d t,
$$

for all $0<s \leq 1$.

Suppose further that $\rho(t)=t^{\mu-1}$ for all $0 \leq t \leq 1$. The following theorem shows that for certain values of $\mu$, the function $g$ in (63) is representable by a convergent Taylor series on the interval $[0,1]$.

Theorem 4.2 Suppose that $0<\alpha<2$ is a real number and $n$ is a positive integer. Then

$$
\begin{gathered}
\pi s^{\frac{2 n-1}{\alpha}-1}+\int_{0}^{1} \frac{t \sin (\pi \alpha)}{s^{2}+t^{2}-2 s t \cos (\pi \alpha)} t^{\frac{2 n-1}{\alpha}-1} d t=\alpha \sum_{m=1}^{\infty} \frac{\sin (m \pi \alpha)}{2 n-1-\alpha m} s^{m-1}, \\
\pi s^{\frac{2 n}{2-\alpha}-1}+\int_{0}^{1} \frac{t \sin (\pi \alpha)}{s^{2}+t^{2}-2 s t \cos (\pi \alpha)} t^{\frac{2 n}{2-\alpha}-1} d t=(2-\alpha) \sum_{m=1}^{\infty} \frac{\sin (m \pi \alpha)}{2 n-(2-\alpha) m} s^{m-1},
\end{gathered}
$$

for all $0<s \leq 1$. 
Proof. Suppose that $\frac{2 n-1}{\alpha}$ is not an integer. Substituting $\mu=\frac{2 n-1}{\alpha}$ into (32), we observe that

$$
\begin{aligned}
& \pi s^{\frac{2 n-1}{\alpha}-1}+\int_{0}^{1} \frac{t^{\frac{2 n-1}{\alpha}} \sin (\pi \alpha)}{s^{2}-2 s t \cos (\pi \alpha)+t^{2}} d t \\
& =\pi s^{\frac{2 n-1}{\alpha}-1}+\pi s^{\frac{2 n-1}{\alpha}-1} \frac{\sin \left(\frac{2 n-1}{\alpha} \cdot \pi(1-\alpha)\right)}{\sin \left(\frac{2 n-1}{\alpha} \cdot \pi\right)}+\sum_{k=0}^{\infty} \frac{\sin ((k+1) \pi \alpha)}{\frac{2 n-1}{\alpha}-k-1} s^{k} \\
& =\pi s^{\frac{2 n-1}{\alpha}-1}+\pi s^{\frac{2 n-1}{\alpha}-1} \frac{\sin \left(\frac{2 n-1}{\alpha} \cdot \pi-(2 n-1) \pi\right)}{\sin \left(\frac{2 n-1}{\alpha} \cdot \pi\right)}+\alpha \sum_{k=0}^{\infty} \frac{\sin ((k+1) \pi \alpha)}{2 n-1-\alpha(k+1)} s^{k} \\
& =\pi s^{\frac{2 n-1}{\alpha}-1}-\pi s^{\frac{2 n-1}{\alpha}-1}+\alpha \sum_{m=1}^{\infty} \frac{\sin (m \pi \alpha)}{2 n-1-\alpha m} s^{m-1} \\
& =\alpha \sum_{m=1}^{\infty} \frac{\sin (m \pi \alpha)}{2 n-1-\alpha m} s^{m-1},
\end{aligned}
$$

for all $s>0$.

Now suppose that $\frac{2 n-1}{\alpha}$ is an integer. We observe that there is a neighborhood $V$ of $\alpha$ such that $\frac{2 n-1}{\alpha}$ is not an integer on $V \backslash\{\alpha\}$. Clearly, (66) is a bounded and analytic on $V \backslash\{\alpha\}$. Therefore, identity (66) is extended to this case by an application of L'Hôpital's rule.

\subsubsection{The Odd Case}

Suppose that $\rho \in L^{2}([-1,1])$ is an odd function, and suppose that $g \in L^{2}([-1,1])$ is defined by (47). By Theorem 4.1, $g$ is also odd and

$$
g(s)=-\pi \rho(s)+\int_{0}^{1} \frac{t \sin (\pi \alpha)}{s^{2}+t^{2}-2 s t \cos (\pi \alpha)} \rho(t) d t,
$$

for all $0<s \leq 1$.

Suppose further that $\rho(t)=t^{\mu-1}$ for all $0 \leq t \leq 1$. The following theorem shows that for certain values of $\mu$, the function $g$ in (67) is representable by a convergent Taylor series on the interval $[0,1]$.

Theorem 4.3 Suppose that $0<\alpha<2$ is a real number and $n$ is a positive integer. 
Then

$$
\begin{gathered}
-\pi s^{\frac{2 n}{\alpha}-1}+\int_{0}^{1} \frac{t \sin (\pi \alpha)}{s^{2}+t^{2}-2 s t \cos (\pi \alpha)} t^{\frac{2 n}{\alpha}-1} d t=\alpha \sum_{m=1}^{\infty} \frac{\sin (m \pi \alpha)}{2 n-\alpha m} s^{m-1} \\
-\pi s^{\frac{2 n-1}{2-\alpha}-1}+\int_{0}^{1} \frac{t \sin (\pi \alpha)}{s^{2}+t^{2}-2 s t \cos (\pi \alpha)} t^{\frac{2 n-1}{2-\alpha}-1} d t=(2-\alpha) \sum_{m=1}^{\infty} \frac{\sin (m \pi \alpha)}{2 n-1-(2-\alpha) m} s^{m-1},
\end{gathered}
$$

for all $0<s \leq 1$.

\subsection{Series Representation of the Solution of Equation (47)}

Suppose that $g$ is a function in $L^{2}([-1,1])$ such that $g_{e}$ and $g_{o}$ defined by $(51)$ and $(54)$ are representable by convergent Taylor series on $[0,1]$. Suppose further that $\rho \in L^{2}([-1,1])$ satisfies equation (47). In this section we observe that $\rho_{e}$ and $\rho_{o}$ defined by (52) and (55) are representable by certain series of singular powers of $x \in[0,1]$.

\subsubsection{The Even Case}

Suppose that $g \in L^{2}([-1,1])$ is an even function, and suppose that $\rho \in L^{2}([-1,1])$ satisfies equation (47). By Theorem 4.1, $\rho$ is also even and

$$
-\pi \rho(s)-\int_{0}^{1} \frac{t \sin (\pi \alpha)}{s^{2}+t^{2}-2 s t \cos (\pi \alpha)} \rho(t) d t=g(s),
$$

for all $0<s \leq 1$, where $0<\alpha<2$.

Let $\lceil x\rceil$ denote the smallest integer $n$ such that $n \geq x$, and let $\lfloor x\rfloor$ denote the largest integer $n$ such that $n \leq x$, for all real $x$. The following theorem shows that if the $g$ is representable by a convergent Taylor series on $[0,1]$, then for any positive integer $n$ there exist unique real numbers $b_{1}, b_{2}, \ldots, b_{n}$ such that the function

$$
\rho(t)=\sum_{i=1}^{\lceil n / 2\rceil} b_{2 i-1} t^{\frac{2 i-1}{\alpha}-1}+\sum_{i=1}^{\lfloor n / 2\rfloor} b_{2 i} t^{\frac{2 i}{2-\alpha}-1},
$$

where $0 \leq t \leq 1$, solves equation (70) to within an error $O\left(t^{n}\right)$.

Theorem 4.4 Suppose that $n$ is a positive integer and $c_{1}, c_{2}, \ldots, c_{n}$ are real numbers. Suppose further that $g:[0,1] \rightarrow \mathbb{R}$ is defined by the formula

$$
g(t)=\sum_{i=1}^{n} c_{i} t^{i-1}
$$


for all $0 \leq t \leq 1$. Then, for all but a finite number of $0<\alpha<2$, there exist unique real numbers $b_{1}, b_{2}, \ldots, b_{n}$ such that

$$
\rho(t)=\sum_{i=1}^{\lceil n / 2\rceil} b_{2 i-1} t^{\frac{2 i-1}{\alpha}-1}+\sum_{i=1}^{\lfloor n / 2\rfloor} b_{2 i} t^{\frac{2 i}{2-\alpha}-1}
$$

for all $0 \leq t \leq 1$, and

$$
-\pi \rho(s)-\int_{0}^{1} \frac{t \sin (\pi \alpha)}{s^{2}+t^{2}-2 s t \cos (\pi \alpha)} \rho(t) d t=g(s)+s^{n} \phi(s),
$$

for all $0<s \leq 1$, where $\phi:[0,1] \rightarrow \mathbb{R}$ is a bounded function representable by a convergent Taylor series of the form

$$
\phi(t)=\sum_{i=1}^{\infty} d_{i} t^{i-1}
$$

for all $0 \leq t \leq 1$, where $d_{1}, d_{2}, \ldots$ are real numbers.

Proof. By Theorem 3.7, the $n \times n$ matrix $A(\alpha)$ defined by (42) is nonsingular for all but a finite number of $0<\alpha<2$. Whenever $A(\alpha)$ is nonsingular, there exist unique real numbers $b_{1}, b_{2}, \ldots, b_{n}$ such that

$$
-\sum_{j=1}^{n} A(\alpha)_{i, j} b_{j}=c_{i}
$$

for every $i=1,2, \ldots, n$. Suppose that $\rho:[0,1] \rightarrow \mathbb{R}$ is defined by (73). By Theorem 4.2,

$$
\begin{aligned}
& -\pi \rho(s)-\int_{0}^{1} \frac{t \sin (\pi \alpha)}{s^{2}+t^{2}-2 s t \cos (\pi \alpha)} \rho(t) d t \\
& =-\alpha \sum_{j=1}^{\lceil n / 2\rceil} b_{2 j-1} \sum_{i=1}^{\infty} \frac{\sin (\pi \alpha i)}{2 j-1-\alpha i} s^{i-1}-(2-\alpha) \sum_{j=1}^{\lfloor n / 2\rfloor} b_{2 j} \sum_{i=1}^{\infty} \frac{\sin (\pi \alpha i)}{2 j-(2-\alpha) i} s^{i-1} \\
& =-\alpha \sum_{j=1}^{\lceil n / 2\rceil} b_{2 j-1} \sum_{i=1}^{n} \frac{\sin (\pi \alpha i)}{2 j-1-\alpha i} s^{i-1}-(2-\alpha) \sum_{j=1}^{\lfloor n / 2\rfloor} b_{2 j} \sum_{i=1}^{n} \frac{\sin (\pi \alpha i)}{2 j-(2-\alpha) i} s^{i-1}+s^{n} \phi(s),
\end{aligned}
$$

for all $0 \leq s \leq 1$, where $\phi:[0,1] \rightarrow \mathbb{R}$ is defined by the formula

$$
\phi(t)=-\alpha \sum_{j=1}^{\lceil n / 2\rceil} b_{2 j-1} \sum_{i=n+1}^{\infty} \frac{\sin (\pi \alpha i)}{2 j-1-\alpha i} t^{i-1-n}-(2-\alpha) \sum_{j=1}^{\lfloor n / 2\rfloor} b_{2 j} \sum_{i=n+1}^{\infty} \frac{\sin (\pi \alpha i)}{2 j-(2-\alpha) i} t^{i-1-n},
$$


for all $0 \leq t \leq 1$. By Theorem 3.6, $\phi$ is bounded on $[0,1]$. By interchanging the order of summation in (77), we observe that

$$
\begin{aligned}
& -\pi \rho(s)-\int_{0}^{1} \frac{t \sin (\pi \alpha)}{s^{2}+t^{2}-2 s t \cos (\pi \alpha)} \rho(t) d t \\
& =\sum_{i=1}^{n}\left(-\alpha \sum_{j=1}^{\lceil n / 2\rceil} \frac{\sin (\pi \alpha i)}{2 j-1-\alpha i} b_{2 j-1}-(2-\alpha) \sum_{j=1}^{\lfloor n / 2\rfloor} \frac{\sin (\pi \alpha i)}{2 j-(2-\alpha) i} b_{2 j}\right) s^{i-1}+s^{n} \phi(s) \\
& =\sum_{i=1}^{n}\left(-\sum_{j=1}^{n} A(\alpha)_{i, j} b_{j}\right) s^{i-1}+s^{n} \phi(s)=\sum_{i=1}^{n} c_{i} s^{i-1}+s^{n} \phi(s)=g(s)+s^{n} \phi(s),
\end{aligned}
$$

for all $0 \leq s \leq 1$

\subsubsection{The Odd Case}

Suppose that $g \in L^{2}([-1,1])$ is an odd function, and suppose that $\rho \in L^{2}([-1,1])$ satisfies equation (47). By Theorem 4.1, $\rho$ is also odd and

$$
-\pi \rho(s)+\int_{0}^{1} \frac{t \sin (\pi \alpha)}{s^{2}+t^{2}-2 s t \cos (\pi \alpha)} \rho(t) d t=g(s),
$$

for all $0<s \leq 1$, where $0<\alpha<2$.

Let $\lceil x\rceil$ denote the smallest integer $n$ such that $n \geq x$, and let $\lfloor x\rfloor$ denote the largest integer $n$ such that $n \leq x$, for all real $x$. The following theorem shows that if the $g$ is representable by a convergent Taylor series on $[0,1]$, then for any positive integer $n$ there exist unique real numbers $b_{1}, b_{2}, \ldots, b_{n}$ such that the function

$$
\rho(t)=\sum_{i=1}^{\lceil n / 2\rceil} b_{2 i-1} t^{\frac{2 i-1}{2-\alpha}-1}+\sum_{i=1}^{\lfloor n / 2\rfloor} b_{2 i} t^{\frac{2 i}{\alpha}-1}
$$

where $0 \leq t \leq 1$, solves equation (80) to within an error $O\left(t^{n}\right)$.

Theorem 4.5 Suppose that $n$ is a positive integer and $c_{1}, c_{2}, \ldots, c_{n}$ are real numbers. Suppose further that $g:[0,1] \rightarrow \mathbb{R}$ is defined by the formula

$$
g(t)=\sum_{i=1}^{n} c_{i} t^{i-1}
$$


for all $0 \leq t \leq 1$. Then, for all but a finite number of $0<\alpha<2$, there exist unique real numbers $b_{1}, b_{2}, \ldots, b_{n}$ so that

$$
\rho(t)=\sum_{i=1}^{\lceil n / 2\rceil} b_{2 i-1} t^{\frac{2 i-1}{2-\alpha}-1}+\sum_{i=1}^{\lfloor n / 2\rfloor} b_{2 i} t^{\frac{2 i}{\alpha}-1}
$$

for all $0 \leq t \leq 1$, and

$$
-\pi \rho(s)+\int_{0}^{1} \frac{t \sin (\pi \alpha)}{s^{2}+t^{2}-2 s t \cos (\pi \alpha)} \rho(t) d t=g(s)+s^{n} \phi(s),
$$

for all $0 \leq s \leq 1$, where $\phi:[0,1] \rightarrow \mathbb{R}$ is a bounded function representable by a convergent Taylor series of the form

$$
\phi(t)=\sum_{i=1}^{\infty} d_{i} t^{i-1}
$$

for all $0 \leq t \leq 1$, where $d_{1}, d_{2}, \ldots$ are real numbers

\subsection{Summary of Results}

We summarize the results of the preceding subsections 4.1, 4.2, 4.3 as follows.

Suppose that the curve $\gamma:[-1,1] \rightarrow \mathbb{R}^{2}$ is a wedge defined by (21) with interior angle $\pi \alpha$, where $0<\alpha<2$ (see Figure 3 ). Let $g \in L^{2}([-1,1])$, and consider the boundary integral equation

$$
-\pi \rho(s)+\int_{-1}^{1} \psi_{\gamma(s), \nu(s)}^{1}(\gamma(t)) \rho(t) d t=g(s),
$$

for all $s \in[-1,1]$, where $\rho \in L^{2}([-1,1])$.

Suppose that the even and odd parts of $g$ are each representable by convergent Taylor series on the interval $[0,1]$. Then, for each positive integer $n$, there exist real numbers $b_{1}, b_{2}, \ldots, b_{n}$ and $c_{1}, c_{2}, \ldots, c_{n}$ such that

$\rho(t)=\sum_{i=1}^{\lceil n / 2\rceil} b_{2 i-1}|t|^{\frac{2 i-1}{\alpha}-1}+\sum_{i=1}^{\lfloor n / 2\rfloor} b_{2 i} \operatorname{sgn}(t)|t|^{\frac{2 i}{\alpha}-1}+\sum_{i=1}^{\lceil n / 2\rceil} c_{2 i-1} \operatorname{sgn}(t)|t|^{\frac{2 i-1}{2-\alpha}-1}+\sum_{i=1}^{\lfloor n / 2\rfloor} c_{2 i}|t|^{\frac{2 i}{2-\alpha}-1}$,

for all $-1 \leq t \leq 1$, solves equation (86) to within an error $O\left(t^{n}\right)$. Moreover, the even and odds parts of this error are also representable by convergent Taylor series on the interval $[0,1]$ (see theorems 4.4 and 4.5 ). 
Observation 4.1 Numerical experiments (see Section 6) suggest that, for a certain subclass of functions $g$, stronger versions of theorems 4.4 and 4.5 are true. Suppose that $G$ is a harmonic function on a neighborhood of the set $\left\{x \in \mathbb{R}^{2}:\|x\| \leq 1\right\}$, and let

$$
g(t)=\frac{\partial G}{\partial \nu(t)}(\gamma(t))
$$

for all $-1 \leq t \leq 1$, where $\nu(t)$ is the inward-pointing unit normal vector at $\gamma(t)$. We conjecture that there exist infinite sequences of real numbers $b_{1}, b_{2}, \ldots$ and $c_{1}, c_{2}, \ldots$ such that

$\rho(t)=\sum_{i=1}^{\infty} b_{2 i-1}|t|^{\frac{2 i-1}{\alpha}-1}+\sum_{i=1}^{\infty} b_{2 i} \operatorname{sgn}(t)|t|^{\frac{2 i}{\alpha}-1}+\sum_{i=1}^{\infty} c_{2 i-1} \operatorname{sgn}(t)|t|^{\frac{2 i-1}{2-\alpha}-1}+\sum_{i=1}^{\infty} c_{2 i}|t|^{\frac{2 i}{2-\alpha}-1}$,

is well defined for all $-1 \leq t \leq 1$, and (89) solves equation (86).

Observation 4.2 Numerical experiments (see Section 6) indicate that the solution to equation (86) is representable by a series of the form (87), to high precision, for a more general class of curves $\gamma$. More specifically, suppose that $\gamma:[-1,1] \rightarrow \mathbb{R}^{2}$ is a wedge in $\mathbb{R}^{2}$ with smooth, curved sides, with a corner at 0 and interior angle $\pi \alpha$. Suppose further

that all derivatives of $\gamma$, 2nd order and higher, approach zero near the corner. Then the solution is representable by a series of the form (87), to high precision.

\section{Analysis of the Integral Equation: the Dirichlet Case}

Suppose that the curve $\gamma:[-1,1] \rightarrow \mathbb{R}^{2}$ is a wedge defined by (21) with interior angle $\pi \alpha$, where $0<\alpha<2$ (see Figure 3 ). Let $g$ be a function in $L^{2}([-1,1])$, and suppose that $\rho \in L^{2}([-1,1])$ solves the equation

$$
g(s)=-\pi \rho(s)+\int_{-1}^{1} \psi_{\gamma(t), \nu(t)}^{1}(\gamma(s)) \rho(t) d t,
$$

for all $s \in[-1,1]$.

In this section, we will analyze this boundary integral equation, which is well-posed even though the curve $\gamma$ is open (see Observation 2.2). In Section 5.1 we investigate the behavior of $(90)$ for functions $\rho \in L^{2}([-1,1])$ of the forms

$$
\begin{aligned}
& \rho(t)=|t|^{\mu}, \\
& \rho(t)=\operatorname{sgn}(t)|t|^{\mu},
\end{aligned}
$$


where $\mu>\frac{1}{2}$ is a real number and

$$
\operatorname{sgn}(x)= \begin{cases}-1 & \text { if } x<0, \\ 0 & \text { if } x=0, \\ 1 & \text { if } x>0,\end{cases}
$$

for all real $x$. If identities (22) and (23) are substituted into (90) and $\rho$ has the forms (91) and (92), then for most values of $\mu$ the resulting $g$ is singular. In Section 5.2, we observe that for certain $\mu$, the function $g$ is smooth. In Section 5.3, we fix $g$ and view (90) as an integral equation in $\rho$. We then observe that for certain classes of functions $g$, the solution $\rho$ is representable by a series of functions of the forms (91) and (92).

The proofs of the theorems in this section are essentially identical to the proofs of the corresponding theorems in Section 4, and are omitted.

\subsection{Integral Equations Near a Corner}

The following lemma uses a symmetry argument to reduce (90) from an integral equation on the interval $[-1,1]$ to two independent integral equations on the interval $[0,1]$.

Theorem 5.1 Suppose that $\rho$ is a function in $L^{2}([-1,1])$ and that $g \in L^{2}([-1,1])$ is given by (90). Suppose further that even functions $g_{e}, \rho_{e} \in L^{2}([-1,1])$ are defined via the formulas

$$
\begin{aligned}
& g_{e}(s)=\frac{1}{2}(g(s)+g(-s)), \\
& \rho_{e}(s)=\frac{1}{2}(\rho(s)+\rho(-s)) .
\end{aligned}
$$

Then

$$
g_{e}(s)=-\pi \rho_{e}(s)-\int_{0}^{1} \frac{s \sin (\pi \alpha)}{s^{2}+t^{2}-2 s t \cos (\pi \alpha)} \rho_{e}(t) d t,
$$

for all $0<s \leq 1$.

Likewise, suppose that odd functions $g_{o}, \rho_{o} \in L^{2}([-1,1])$ are defined via the formulas

$$
\begin{aligned}
& g_{o}(s)=\frac{1}{2}(g(s)-g(-s)), \\
& \rho_{o}(s)=\frac{1}{2}(\rho(s)-\rho(-s)) .
\end{aligned}
$$

Then

$$
g_{o}(s)=-\pi \rho_{o}(s)+\int_{0}^{1} \frac{s \sin (\pi \alpha)}{s^{2}+t^{2}-2 s t \cos (\pi \alpha)} \rho_{o}(t) d t,
$$

for all $0<s \leq 1$. 


\subsection{The Singularities in the Solution of Equation (90)}

In this section we observe that for certain functions $\rho$, the functions $g_{e}$ and $g_{o}$ defined by (94) and (97) are representable by convergent Taylor series on $[0,1]$.

\subsubsection{The Even Case}

Suppose that $\rho \in L^{2}([-1,1])$ is an even function, and suppose that $g \in L^{2}([-1,1])$ is defined by (90). By Theorem 5.1, $g$ is also even and

$$
g(s)=-\pi \rho(s)-\int_{0}^{1} \frac{s \sin (\pi \alpha)}{s^{2}+t^{2}-2 s t \cos (\pi \alpha)} \rho(t) d t,
$$

for all $0<s \leq 1$.

Suppose further that $\rho(t)=t^{\mu-1}$ for all $0 \leq t \leq 1$. The following theorem shows that for certain values of $\mu$, the function $g$ in (100) is representable by a convergent Taylor series on the interval $[0,1]$.

Theorem 5.2 Suppose that $0<\alpha<2$ is a real number and $n$ is a positive integer. Then

$$
\begin{gathered}
\pi+\int_{0}^{1} \frac{s \sin (\pi \alpha)}{s^{2}+t^{2}-2 s t \cos (\pi \alpha)} d t=(2-\alpha) \pi-\sum_{m=1}^{\infty} \frac{\sin (m \pi \alpha)}{m} s^{m}, \\
\pi s^{\frac{2 n-1}{\alpha}}+\int_{0}^{1} \frac{s \sin (\pi \alpha)}{s^{2}+t^{2}-2 s t \cos (\pi \alpha)} t^{\frac{2 n-1}{\alpha}} d t=\alpha \sum_{m=1}^{\infty} \frac{\sin (m \pi \alpha)}{2 n-1-\alpha m} s^{m}, \\
\pi s^{\frac{2 n}{2-\alpha}}+\int_{0}^{1} \frac{s \sin (\pi \alpha)}{s^{2}+t^{2}-2 s t \cos (\pi \alpha)} t^{\frac{2 n}{2-\alpha}} d t=(2-\alpha) \sum_{m=1}^{\infty} \frac{\sin (m \pi \alpha)}{2 n-(2-\alpha) m} s^{m},
\end{gathered}
$$

for all $0<s \leq 1$.

Proof. Taking the limit $\mu \rightarrow 0$ in (32) and applying L'Hôpital's rule once, we observe that

$$
\int_{0}^{1} \frac{\sin (\pi \alpha)}{a^{2}-2 a x \cos (\pi \alpha)+x^{2}} d x=(1-\alpha) \pi a^{-1}-\sum_{k=0}^{\infty} \frac{\sin ((k+1) \pi \alpha)}{k+1} a^{k}
$$

for all $0<a \leq 1$, from which identity (101) clearly follows.

The proofs of identities (102) and (103) are essentially identical to the corresponding proofs in Theorem 4.2 . 


\subsubsection{The Odd Case}

Suppose that $\rho \in L^{2}([-1,1])$ is an odd function, and suppose that $g \in L^{2}([-1,1])$ is defined by (47). By Theorem 4.1, $g$ is also odd and

$$
g(s)=-\pi \rho(s)+\int_{0}^{1} \frac{t \sin (\pi \alpha)}{s^{2}+t^{2}-2 s t \cos (\pi \alpha)} \rho(t) d t,
$$

for all $0<s \leq 1$.

Suppose further that $\rho(t)=t^{\mu-1}$ for all $0 \leq t \leq 1$. The following theorem shows that for certain values of $\mu$, the function $g$ in (67) is representable by a convergent Taylor series on the interval $[0,1]$.

Theorem 5.3 Suppose that $0<\alpha<2$ is a real number and $n$ is a positive integer. Then

$$
\begin{gathered}
-\pi+\int_{0}^{1} \frac{s \sin (\pi \alpha)}{s^{2}+t^{2}-2 s t \cos (\pi \alpha)} d t=-\alpha \pi-\sum_{m=1}^{\infty} \frac{\sin (m \pi \alpha)}{m} s^{m}, \\
-\pi s^{\frac{2 n}{\alpha}}+\int_{0}^{1} \frac{s \sin (\pi \alpha)}{s^{2}+t^{2}-2 s t \cos (\pi \alpha)} t^{\frac{2 n}{\alpha}} d t=\alpha \sum_{m=1}^{\infty} \frac{\sin (m \pi \alpha)}{2 n-\alpha m} s^{m}, \\
-\pi s^{\frac{2 n-1}{2-\alpha}}+\int_{0}^{1} \frac{s \sin (\pi \alpha)}{s^{2}+t^{2}-2 s t \cos (\pi \alpha)} t^{\frac{2 n-1}{2-\alpha}} d t=(2-\alpha) \sum_{m=1}^{\infty} \frac{\sin (m \pi \alpha)}{2 n-1-(2-\alpha) m} s^{m},
\end{gathered}
$$

for all $0<s \leq 1$.

\subsection{Series Representation of the Solution of Equation (90)}

Suppose that $g$ is a function in $L^{2}([-1,1])$ such that $g_{e}$ and $g_{o}$ defined by $(51)$ and $(54)$ are representable by convergent Taylor series on $[0,1]$. Suppose further that $\rho \in L^{2}([-1,1])$ satisfies equation (47). In this section we observe that $\rho_{e}$ and $\rho_{o}$ defined by (52) and (55) are representable by certain series of singular powers of $x \in[0,1]$.

\subsubsection{The Even Case}

Suppose that $g \in L^{2}([-1,1])$ is an even function, and suppose that $\rho \in L^{2}([-1,1])$ satisfies equation (47). By Theorem 4.1, $\rho$ is also even and

$$
-\pi \rho(s)-\int_{0}^{1} \frac{s \sin (\pi \alpha)}{s^{2}+t^{2}-2 s t \cos (\pi \alpha)} \rho(t) d t=g(s),
$$


for all $0<s \leq 1$, where $0<\alpha<2$.

Let $\lceil x\rceil$ denote the smallest integer $n$ such that $n \geq x$, and let $\lfloor x\rfloor$ denote the largest integer $n$ such that $n \leq x$, for all real $x$. The following theorem shows that if the $g$ is representable by a convergent Taylor series on $[0,1]$, then for any positive integer $n$ there exist unique real numbers $b_{0}, b_{1}, \ldots, b_{n}$ such that the function

$$
\rho(t)=\sum_{i=1}^{\lceil n / 2\rceil} b_{2 i-1} t^{\frac{2 i-1}{\alpha}}+\sum_{i=0}^{\lfloor n / 2\rfloor} b_{2 i} t^{\frac{2 i}{2-\alpha}}
$$

where $0 \leq t \leq 1$, solves equation (109) to within an error $O\left(t^{n+1}\right)$.

Theorem 5.4 Suppose that $n$ is a positive integer and $c_{0}, c_{1}, \ldots, c_{n}$ are real numbers. Suppose further that $g:[0,1] \rightarrow \mathbb{R}$ is defined by the formula

$$
g(t)=\sum_{i=0}^{n} c_{i} t^{i}
$$

for all $0 \leq t \leq 1$. Then, for all but a finite number of $0<\alpha<2$, there exist unique real numbers $b_{0}, b_{1}, \ldots, b_{n}$ so that

$$
\rho(t)=\sum_{i=1}^{\lceil n / 2\rceil} b_{2 i-1} t^{\frac{2 i-1}{\alpha}}+\sum_{i=0}^{\lfloor n / 2\rfloor} b_{2 i} t^{\frac{2 i}{2-\alpha}}
$$

for all $0 \leq t \leq 1$, and

$$
-\pi \rho(s)-\int_{0}^{1} \frac{t \sin (\pi \alpha)}{s^{2}+t^{2}-2 s t \cos (\pi \alpha)} \rho(t) d t=g(s)+s^{n+1} \phi(s),
$$

for all $0<s \leq 1$, where $\phi:[0,1] \rightarrow \mathbb{R}$ is a bounded function representable by a convergent Taylor series of the form

$$
\phi(t)=\sum_{i=0}^{\infty} d_{i} t^{i}
$$

for all $0 \leq t \leq 1$, where $d_{0}, d_{1}, \ldots$ are real numbers

\subsubsection{The Odd Case}

Suppose that $g \in L^{2}([-1,1])$ is an odd function, and suppose that $\rho \in L^{2}([-1,1])$ satisfies equation (47). By Theorem 4.1, $\rho$ is also odd and

$$
-\pi \rho(s)+\int_{0}^{1} \frac{s \sin (\pi \alpha)}{s^{2}+t^{2}-2 s t \cos (\pi \alpha)} \rho(t) d t=g(s),
$$


for all $0<s \leq 1$, where $0<\alpha<2$.

Let $\lceil x\rceil$ denote the smallest integer $n$ such that $n \geq x$, and let $\lfloor x\rfloor$ denote the largest integer $n$ such that $n \leq x$, for all real $x$. The following theorem shows that if the $g$ is representable by a convergent Taylor series on $[0,1]$, then for any positive integer $n$ there exist unique real numbers $b_{0}, b_{1}, \ldots, b_{n}$ such that the function

$$
\rho(t)=\sum_{i=1}^{\lceil n / 2\rceil} b_{2 i-1} t^{\frac{2 i-1}{2-\alpha}}+\sum_{i=0}^{\lfloor n / 2\rfloor} b_{2 i} t^{\frac{2 i}{\alpha}}
$$

where $0 \leq t \leq 1$, solves equation (115) to within an error $O\left(t^{n+1}\right)$.

Theorem 5.5 Suppose that $n$ is a positive integer and $c_{0}, c_{1}, \ldots, c_{n}$ are real numbers. Suppose further that $g:[0,1] \rightarrow \mathbb{R}$ is defined by the formula

$$
g(t)=\sum_{i=0}^{n} c_{i} t^{i}
$$

for all $0 \leq t \leq 1$. Then, for all but a finite number of $0<\alpha<2$, there exist unique real numbers $b_{0}, b_{1}, \ldots, b_{n}$ so that

$$
\rho(t)=\sum_{i=1}^{\lceil n / 2\rceil} b_{2 i-1} t^{\frac{2 i-1}{2-\alpha}}+\sum_{i=0}^{\lfloor n / 2\rfloor} b_{2 i} t^{\frac{2 i}{\alpha}}
$$

for all $0 \leq t \leq 1$, and

$$
-\pi \rho(s)+\int_{0}^{1} \frac{t \sin (\pi \alpha)}{s^{2}+t^{2}-2 s t \cos (\pi \alpha)} \rho(t) d t=g(s)+s^{n+1} \phi(s),
$$

for all $0<s \leq 1$, where $\phi:[0,1] \rightarrow \mathbb{R}$ is a bounded function representable by a convergent Taylor series of the form

$$
\phi(t)=\sum_{i=0}^{\infty} d_{i} t^{i}
$$

for all $0 \leq t \leq 1$, where $d_{0}, d_{1}, \ldots$ are real numbers.

\subsection{Summary of Results}

We summarize the results of the preceding subsections $5.1,5.2,5.3$ as follows. 
Suppose that the curve $\gamma:[-1,1] \rightarrow \mathbb{R}^{2}$ is a wedge defined by (21) with interior angle $\pi \alpha$, where $0<\alpha<2$ (see Figure 3 ). Let $g \in L^{2}([-1,1])$, and consider the boundary integral equation

$$
\pi \rho(s)+\int_{-1}^{1} \psi_{\gamma(s), \nu(s)}^{1}(\gamma(t)) \rho(t) d t=g(s)
$$

for all $s \in[-1,1]$, where $\rho \in L^{2}([-1,1])$.

Suppose that the even and odd parts of $g$ are each representable by convergent Taylor series on the interval $[0,1]$. Then, for each positive integer $n$, there exist real numbers $b_{0}, b_{1}, \ldots, b_{n}$ and $c_{0}, c_{1}, \ldots, c_{n}$ such that

$$
\rho(t)=\sum_{i=1}^{\lceil n / 2\rceil} b_{2 i-1}|t|^{\frac{2 i-1}{\alpha}}+\sum_{i=0}^{\lfloor n / 2\rfloor} b_{2 i} \operatorname{sgn}(t)|t|^{\frac{2 i}{\alpha}}+\sum_{i=1}^{\lceil n / 2\rceil} c_{2 i-1} \operatorname{sgn}(t)|t|^{\frac{2 i-1}{2-\alpha}}+\sum_{i=0}^{\lfloor n / 2\rfloor} c_{2 i}|t|^{\frac{2 i}{2-\alpha}}
$$

for all $-1 \leq t \leq 1$, solves equation (121) to within an error $O\left(t^{n+1}\right)$. Moreover, the even and odds parts of this error are also representable by convergent Taylor series on the interval $[0,1]$ (see theorems 5.4 and 5.5).

Observation 5.1 Numerical experiments (see Section 6) suggest that, for a certain subclass of functions $g$, stronger versions of theorems 5.4 and 5.5 are true. Suppose that $G$ is a harmonic function on a neighborhood of the set $\left\{x \in \mathbb{R}^{2}:\|x\| \leq 1\right\}$, and let

$$
g(t)=G(\gamma(t))
$$

for all $-1 \leq t \leq 1$. We conjecture that there exist infinite sequences of real numbers $b_{0}, b_{1}, \ldots$ and $c_{0}, c_{1}, \ldots$ such that

$\rho(t)=\sum_{i=1}^{\infty} b_{2 i-1}|t|^{\frac{2 i-1}{\alpha}}+\sum_{i=0}^{\infty} b_{2 i} \operatorname{sgn}(t)|t|^{\frac{2 i}{\alpha}}+\sum_{i=1}^{\infty} c_{2 i-1} \operatorname{sgn}(t)|t|^{\frac{2 i-1}{2-\alpha}}+\sum_{i=0}^{\infty} c_{2 i}|t|^{\frac{2 i}{2-\alpha}}$,

is well defined for all $-1 \leq t \leq 1$, and (124) solves equation (121).

Observation 5.2 Numerical experiments (see Section 6) indicate that the solution to equation (121) is representable by a series of the form (122), to high precision, for a more general class of curves $\gamma$. More specifically, suppose that $\gamma:[-1,1] \rightarrow \mathbb{R}^{2}$ is a wedge in $\mathbb{R}^{2}$ with smooth, curved sides, with a corner at 0 and interior angle $\pi \alpha$. Suppose further that all derivatives of $\gamma$, 2nd order and higher, approach zero near the corner. Then the solution is representable by a series of the form (122), to high precision. 


\section{The Algorithm}

To solve the integral equations of potential theory on polygonal domains, we use the algorithm described in [3]; however, instead of discretizing the corner singularities using nested quadratures, we use the representations (87), (122) to construct purpose-made discretizations (see, for example, [19], [17], [25]). A detailed description of this part of the procedure will be published at a later date.

We illustrate the performance of the algorithm with several numerical examples. The exterior Neumann problem and interior Dirichlet problem were solved on each of the domains in figures $4-9$, where the boundary data was generated by a unit charge inside the region in the Neumann case, and outside the region in the Dirichlet case. The numerical solution was tested by comparing the computed potential to the true potential at five arbitrary points. Tables 1 and 2 present the results. The following quantities are reported for each problem:

$n$ is the total number of nodes;

$t$ is the wall clock time required to solve the problem;

$E$ is the largest error in absolute value measured in the computed potential;

$\kappa$ is the condition number of the linear system that was solved.

Observation 6.1 Clearly, the curves $\Gamma_{1}$ and $\Gamma_{2}$ in figures 4 and 5 are not polygons. However, all derivatives of the curves, 2nd order and higher, approach zero near the corners. We observe that in this case, the singularities in the solutions of the boundary integral equations are identical to those in the polygonal case.

Observation 6.2 We observe that if the boundary values are produced by a charge inside the domain in the Neumann case, or outside the domain in the Dirichlet case, certain terms in the representations of the solutions near a corner vanish. More specifically, in the Neumann case, the terms $c_{1}, c_{2}, \ldots$ in (89) vanish, while in the Dirichlet case, the terms $b_{0}, b_{1}, \ldots$ in (124) vanish.

Observation 6.3 The purpose of the numerical examples in this section is to illustrate the accuracy and convergence of the algorithms, as opposed to the resulting CPU time. For historical reasons, the resulting linear systems were solved using the minimal residual method (see, for example, [8]). Clearly, the CPU times in tables 1 and 2 would be significantly improved by using an algorithm appropriate to the size of the problems being solved. 


\section{Extensions and Generalizations}

\subsection{The Helmholtz Equation on Polygonal Domains}

In this paper, we investigate the solution of boundary value problems for Laplace's equation on polygonal domains; we observe that, when the problems are formulated as the boundary integral equations of classical potential theory, the solutions are representable by series of elementary functions. A similar analysis applies to the Helmholtz equation on polygonal domains. More specifically, if boundary value problems for the Helmholtz equation on polygonal domains are formulated as the boundary integral equations of classical potential theory, the solutions are representable by series of appropriately selected Bessel functions. A paper containing this analysis (together with the requisite numerical apparatus) is in preparation.

\subsection{Curved Boundaries with Corners}

While this paper only deals with the solution of Laplace's equation on domains with polygonal boundaries, a similar analysis applies to the case of curved boundaries with corners. More specifically, if the boundary is smooth except at corners, the solutions to the associated boundary integral equations of classical potential theory are also representable by series of elementary functions. This analysis, along with the requisite numerical apparatus, will be described in a forthcoming paper.

\subsection{Generalization to Three Dimensions}

The generalization of the apparatus of this paper to three dimensions is fairly straightforward, but the detailed analysis has not been carried out. This line of research is being vigorously pursued.

\subsection{Robin and Mixed Boundary Conditions}

This paper deals with the solution of Laplace's equation on polygonal domains with either Dirichlet or Neumann boundary conditions. There are two additional boundary conditions that have not yet been analyzed in detail: the Robin condition, which specifies a linear combination of the values of the solution and the values of its derivative on the boundary; and the mixed boundary condition, which specifies Dirichlet boundary conditions on some sides of the polygon and Neumann boundary conditions on others. The results of our pending investigation will be reported at a later date. 


\begin{tabular}{lllll} 
& $n$ & $t$ & $E$ & $\kappa$ \\
\hline$\Gamma_{1}$ & 180 & 0.041426 & $9.88098491916389 \times 10^{-15}$ & 4.7357 \\
$\Gamma_{2}$ & 228 & 0.086169 & $4.13558076672871 \times 10^{-15}$ & 6.1593 \\
$\Gamma_{3}$ & 219 & 0.033944 & $9.99200722162641 \times 10^{-16}$ & 7.5586 \\
$\Gamma_{4}$ & 289 & 0.064797 & $3.60822483003176 \times 10^{-15}$ & 18.363 \\
$\Gamma_{5}$ & 704 & 1.428900 & $5.55111512312578 \times 10^{-16}$ & 21.365 \\
$\Gamma_{6}$ & 1007 & 4.284600 & $1.22124532708767 \times 10^{-15}$ & 27.666 \\
\hline
\end{tabular}

Table 1: Numerical results for the exterior Neumann problem

\begin{tabular}{lllll} 
& $n$ & $t$ & $E$ & $\kappa$ \\
\hline$\Gamma_{1}$ & 162 & 0.078327 & $4.88498130835069 \times 10^{-15}$ & 28.640 \\
$\Gamma_{2}$ & 250 & 0.075677 & $1.33226762955019 \times 10^{-14}$ & 5.4339 \\
$\Gamma_{3}$ & 303 & 0.046949 & $3.05311331771918 \times 10^{-16}$ & 31.302 \\
$\Gamma_{4}$ & 349 & 0.068338 & $5.21804821573824 \times 10^{-15}$ & 107.14 \\
$\Gamma_{5}$ & 776 & 0.425080 & $2.86437540353290 \times 10^{-14}$ & 99.830 \\
$\Gamma_{6}$ & 997 & 0.781490 & $1.08801856413265 \times 10^{-14}$ & 46.737 \\
\hline
\end{tabular}

Table 2: Numerical results for the interior Dirichlet problem 


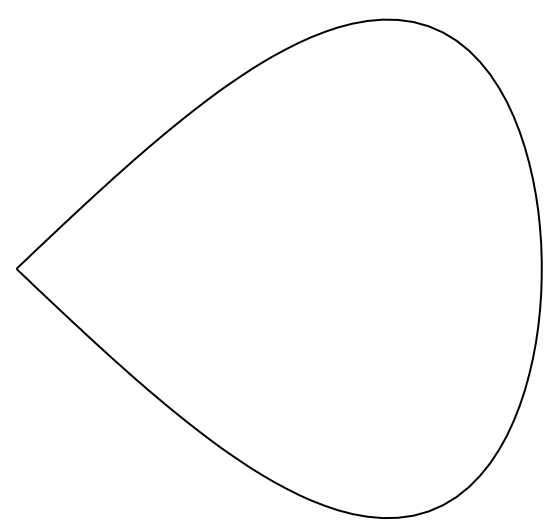

Figure 4: A cone $\Gamma_{1}$ in $\mathbb{R}^{2}$

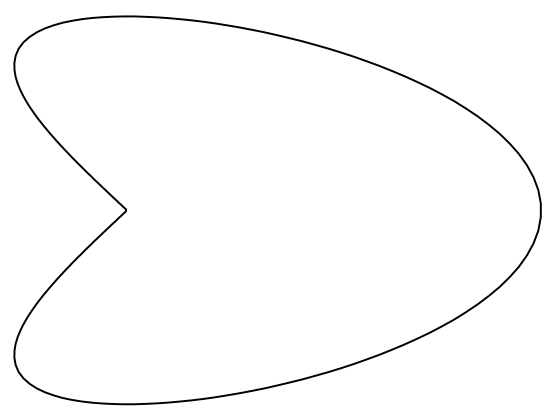

Figure 5: A curve $\Gamma_{2}$ in $\mathbb{R}^{2}$ with an inward-pointing wedge 


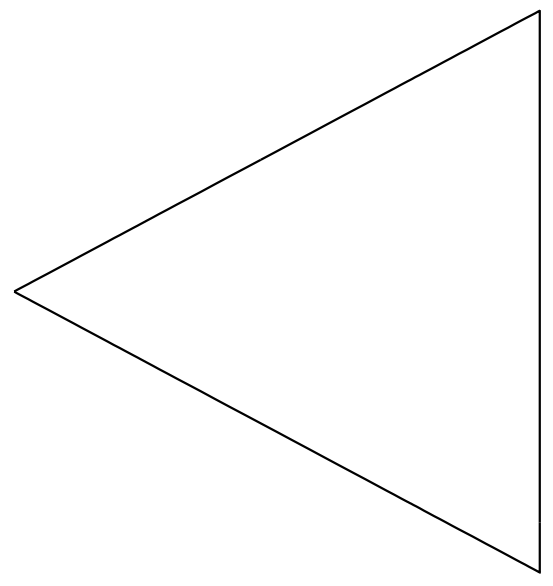

Figure 6: An equilateral triangle $\Gamma_{3}$ in $\mathbb{R}^{2}$

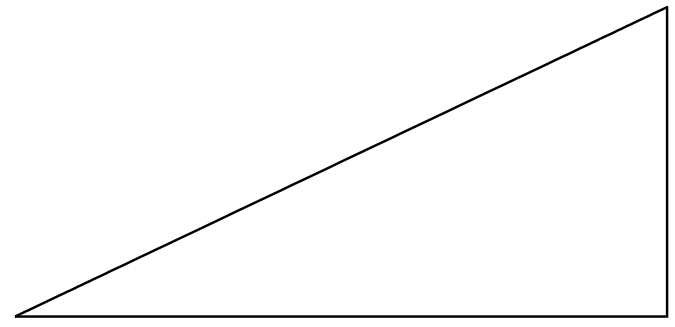

Figure 7: A right triangle $\Gamma_{4}$ in $\mathbb{R}^{2}$

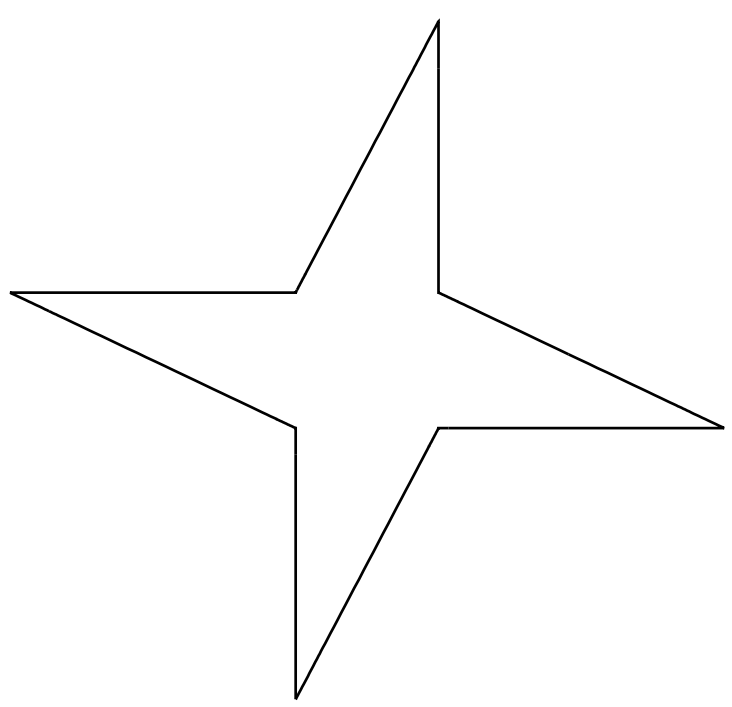

Figure 8: A star-shaped curve $\Gamma_{5}$ in $\mathbb{R}^{2}$ 


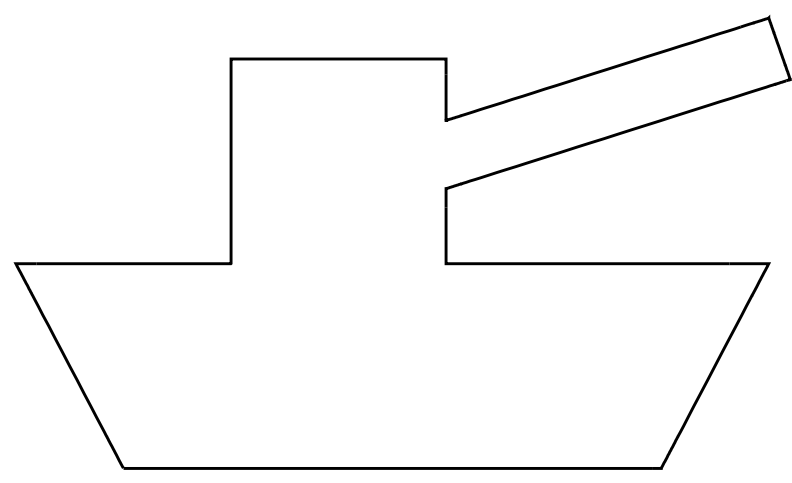

Figure 9: A tank-shaped curve $\Gamma_{6}$ in $\mathbb{R}^{2}$ 


\section{References}

[1] Abramowitz, Milton, and Irene A. Stegun, eds. Handbook of Mathematical Functions With Formulas, Graphs, and Mathematical Tables. Washington: U.S. Govt. Print. Off., 1964.

[2] Apostol, Tom M. Mathematical Analysis. New York: Pearson, 1974.

[3] Bremer, James. "A fast direct solver for the integral equations of scattering theory on planar curves with corners." J. Comput. Phys. 231 (2012): 1879-1899.

[4] Calderón, A. P., C. P. Calderón, E. Fabes, M. Jodeit, and N. M. Riviere. "Applications of the Cauchy Integral on Lipschitz Curves." B. Am. Math. Soc. 84.2 (1978): $287-290$.

[5] Carleson, L. "On the Support of Harmonic Measure for Sets of Cantor Type." Ann. Acad. Sci. Fenn. 10 (1985): 113-123.

[6] Coifman, R. R., Peter W. Jones, and Stephen Semmes. "Two elementary Proofs of the $L^{2}$ Boundedness of Cauchy Integrals on Lipschitz Curves." J. Am. Math. Soc. 2.3 (1989): 553-564.

[7] Courant, R. and D. Hilbert. Methods of Mathematical Physics. New York: Interscience Publishers, 1966.

[8] Golub, Gene H. and Charles F. Van Loan. Matrix Computations. 4th ed. Baltimore: The Johns Hopkins University Press, 2013.

[9] Gradshteyn, I. S., and I. M. Ryzhik. Table of Integrals, Series, and Products. 7th ed. Eds. Alan Jeffrey and Daniel Zwillinger. San Diego: Academic Press, 2000.

[10] Greengard, Leslie, and Vladimir Rokhlin. "A fast algorithm for particle simulations." J. Comput. Phys. 73.2 (1987): 325-348.

[11] Grisvard, Pierre. Elliptic problems in nonsmooth domains. Boston: Pitman Advanced Pub. Program, 1985.

[12] Hao, S., A. H. Barnett, P. G. Martinsson, and P. Young. "High-order accurate Nyström discretization of integral equations with weakly singular kernels on smooth curves in the plane." it Adv. Comput. Math. 40 (2014)

[13] Helsing, J. and R. Ojala. "Corner singularities for elliptic problems: integral equations, graded meshes, quadrature, and compressed inverse preconditioning." J. Comput. Phys. 227 (2008): 8820-8840. 
[14] Jerison, David S., and Carlos E. Kenig. "The Neumann Problem on Lipschitz Domains." B. Am. Math. Soc. 4.2 (1981): 203-207.

[15] Jones, P., J. Ma, and V. Rokhlin. "A fast direct algorithm for the solution of the Laplace equation on regions with fractal boundaries." J. Comput. Phys. 113.1 (1994): 35-51.

[16] Lehman, R. Sherman. "Development of the Mapping Function at an Analytic Corner." Pacific Journal of Mathematics 7.3 (1957): 1437-1449.

[17] Ma, J., V. Rokhlin, and S. Wandzura. "Generalized Gaussian Quadrature Rules for Systems of Arbitrary Functions." SIAM. J. Numer. Anal. 33.3 (1996): 971-996.

[18] Markushevich, A. I. Complex analysis. 2nd ed. Trans. Richard A. Silverman. New York: Chelsea Publishing, 1977.

[19] Martinsson, Per-Gunnar, Vladimir Rokhlin, and Mark Tygert. "On Interpolation and Integration in Finite-Dimensional Spaces of Bounded Functions." Comm. App. Math. and Comp. Sci. 1.1 (2006): 133-142.

[20] Maz'ya, V. G. and S. M. Nikol'skii, eds. Analysis IV: Linear and Boundary Integral Equations (Encycopedia of Mathematical Sciences). Trans. A. Böttcher and S. Prössdorf. New York: Springer-Verlag, 1991.

[21] Petrovsky, I. G. Lectures on Partial Differential Equations. 4th printing. New York: Interscience Publishers, 1954.

[22] Rokhlin, V. "Rapid Solution of Integral Equations of Classical Potential Theory." J. Comput. Phys. 60 (1983): 187-207.

[23] Trefethen, Lloyd N. "Numerical Computation of the Schwarz-Christoffel Transformation." SIAM J. Sci. Stat. Comput. 1.1 (1980): 82-102.

[24] Verchota, Gregory. "Layer Potentials and Regularity for the Dirichlet Problem for Laplace's equation in Lipschitz Domains." J. Funct. Anal. 59 (1984): 572-611.

[25] Yarvin, N. and V. Rokhlin. "Generalized Gaussian Quadratures and Singular Value Decompositions of Integral Operators." SIAM J. Sci. Comput. 20.2 (1998): 699-718.

[26] Zargaryan, S. S., and V. G. Maz'ya. "The Asymptotic Form of the Solutions of the Integral Equations of Potential Theory in the Neighborhood of the Corner Points of a Contour." PMM U.S.S.R. 48.1 (1984): 120-124. 